\title{
Reabilitação protética da maxila atrésica e da disfunção velofaríngea: relato de caso clínico
}

\author{
José Fernando Scarelli LOPES ${ }^{1}$; Ana Luisa Lopes RABELO²; Mônica \\ Moraes Waldemarin LOPES ${ }^{3}$; Alexia Medeiros AMARAL4; Maria \\ Daniela Borro PINT05; Melina Evangelista WHITAKER ${ }^{5}$
}

1 - Cirurgiẫo-dentista, Doutor em Fissuras Orofaciais e Anomalias Relacionadas, Departamento de Prótese Dentária, Hospital de Reabilitação de Anomalias Craniofaciais, Universidade de São Paulo; 2 - Cirurgiã-dentista, Especialista em Prótese Dentária, Hospital de Reabilitação de Anomalias Craniofaciais, Universidade de São Paulo; 3 - Cirurgiâ-dentista, Doutora em Fissuras Orofaciais e Anomalias Relacionadas, Departamento de Prótese Dentária, Hospital de Reabilitação de Anomalias Craniofaciais, Universidade de São Paulo; 4 - Fonoaudióloga, Aluna do Programa de Residência Multiprofissional em Síndromes e Anomalias Craniofaciais, Hospital de Reabilitação de Anomalias Craniofaciais, Universidade de São Paulo; 5 - Fonoaudióloga, Doutora em Ciências da Reabilitação, Serviço de Prótese de Palato, Hospital de Reabilitação de Anomalias Craniofaciais, Universidade de São Paulo.

\section{Resumo}

A fissura de lábio e palato envolve a região do rebordo alveolar, podendo ocasionar desenvolvimento anômalo dos dentes, na região da falha que alteram o alinhamento dentário, comprometendo a estética do sorriso. Além disso, as cirurgias para sua correção podem apresentar efeitos restritivos no desenvolvimento da maxila trazendo alterações no terço médio da face, incluindo a atresia maxilar. Esta pode acarretar em instabilidade oclusal, estética insatisfatória, alterações fonéticas e respiração bucal. Uma vez que as cirurgias de correção da fissura de palato não tenham sido completamente bem sucedidas, o indivíduo pode apresentar uma disfunção velofaríngea. Esta compromete o equilíbrio da ressonância entre as cavidades nasal e oral, ocasionando alterações na fala como: compensações articulatórias e hipernasalidade. A proposta deste trabalho é descrever a reabilitação protética de um paciente, do sexo masculino, 26 anos de idade, com fissura transforame incisivo bilaterale diagnósticos de atresia maxilar e disfunção velofaríngea, devido às sequelas pós-cirúrgicas. Com o objetivo de reabilitar a estética e as funções orais deste indivíduo, foi confeccionada uma prótese parcial removível de recobrimento, retida por coroas metálicas fresadas, nos dentes 23 e 26 , e coroas metálicas nos dentes 13 e 16, unidas por uma barra e uso de sistema barra-clip, para melhorar sua retenção. Para a reabilitação de fala, foi proposto um obturador faríngeo associado à prótese dentária. Concluise que este tratamento proporcionou melhora da sua estética, bem como as funções alimentação e fala, proporcionando, assim, melhora da autoestima e qualidade de vida do indivíduo.

Copyright $(\odot 2021$ Revista

PALAVRAS-CHAVE: Fenda labial. Fenda palatina. Insuficiência Velofaríngea.

\section{AUTOR PARA CORRESPONDÊNCIA}

Jose Fernando Scarelli Lopes

Departamento de Prótese Dentária, Hospital de Reabilitação de Anomalias Craniofaciais HRAC-USP, Bauru,

São Paulo, Cep.:17012-900, Brasil

Telefone: (14) 997938054 / E-mail: jscarelli@usp.br 


\section{Introdução}

As fissuras labiopalatinas são as malformações congênitas craniofaciais mais comuns e ocorrem com uma frequência de 1 a cada 757,5 nascimentos e em 1 a cada 923,53 nascido-vivos. Quando há falta de fechamento de processos faciais, durante o período embrionário, as fissuras labiopalatinas podem acarretar em alterações do sistema estomatognático, com prejuízos estéticos e funcionais de graus variados ${ }^{1-3}$. Quando completa, a fissura de lábio e palato, também chamada de transforame incisivo, envolve a região do rebordo alveolar, ocasionando um desenvolvimento anômalo dos dentes, na região da falha, ou até mesmo, agenesias que alteram o alinhamento dentário, comprometendo a estética do sorriso. Além disso, as cirurgias realizadas para correção da fissura labiopalatina, tanto do lábio quanto do palato, durante a fase de crescimento facial, apesar de objetivarem a reabilitação estética e funcional, podem apresentar efeitos restritivos no desenvolvimento contínuo da maxila ${ }^{4-9}$ trazendo alterações no terço médio da face. Desta forma, em indivíduos com fissura labiopalatina operados, pode haver deficiência transversal da maxila, discrepâncias entre os arcos dentários, além de alteração na dimensão vertical e na oclusão ${ }^{5}$.

Para a correção dessas discrepâncias maxilomandibulares, geralmente, são indicados tratamentos de ortodontia e cirurgia ortognática. Porém, dependendo da evolução do caso, pode haver indicação de próteses parciais removíveis de recobrimento “overlay”, que podem proporcionar melhora da estética facial, devido á recuperação da oclusão e do suporte labial4,10. A indicação do tratamento deve ser analisada pela equipe interdisciplinar, apoiado na limitação de cada caso.

Em relação à complexidade do tratamento de indivíduos com fissura labiopalatina, além do comprometimento do desenvolvimento facial, ocasionado pelas cirurgias primárias, pode haver também alterações no fechamento velofaríngeo. Isso porque para a produção de sons orais da fala é necessária a sincronia 
entre palato mole, paredes laterais e parede posterior da faringe, que são as estruturas responsáveis pela distribuição e orientação do fluxo de ar expiratório e das vibrações acústicas, para a cavidade oral ${ }^{2,12}$. Com isso, mesmo após a cirurgia de palatoplastia, alguns indivíduos permanecem com a disfunção velofaríngea, sendo esta um alteração anatômica e/ou funcional deste mecanismo, prejudicando assim a inteligibilidade de fala ${ }^{11,2}$. Como tratamento da disfunção velofaríngea, mais uma vez, a equipe interdisciplinar deve analisar as limitações de cada caso, optando por indicar um novo procedimento cirúrgico para reabilitação do mecanismo velofaríngeo, ou ainda, a confecção de um obturador faríngeo, ambos associados à fonoterapia ${ }^{13,14}$.

Quando a indicação para a melhora oclusal é um prótese removível, geralmente é indicado um obturador faríngeo, associado a esta prótese dentária, com o objetivo de reabilitar a disfunção velofaríngea. O obturador faríngeo é caracterizado por uma extensão acrílica posicionada na região da nasofaringe, que tem a função de obturar o gap velofaríngeo, isto é, a falha de fechamento das estruturas envolvidas para a produção correta de sons orais ${ }^{15}$. A prótese com obturador faríngeo pode possibilitar a melhora da fala do paciente, uma vez que auxilia na terapia fonoaudiológica. O papel do fonoaudiólogo é importante em todas as etapas do tratamento: desde o diagnóstico da disfunção velofaríngea, definição de conduta para reabilitação desta, confecção do obturador faríngeo e controle da prótese para acompanhamento da alimentação e da fala ${ }^{16}$.

Contudo, a proposta deste trabalho é descrever a reabilitação oral de um paciente com fissura de labio e palato bilateral operado, com dimensão vertical de oclusão reduzida, maxila atrésica, sem indicação oportuna de ortodontia e cirurgia ortognática e com disfunção velofaríngea, por meio da confecção de uma prótese parcial removível de recobrimento "overlay" associada ao obturador faríngeo. 


\section{Relato de caso}

Paciente do sexo masculino, de 26 anos de idade, com fissura transforame bilateral, com histórico de ter sido submetido à cirurgia de lábio, palato duro, palato mole e outras cirurgias plásticas em um hospital de referência em Síndromes e Anomalias Craniofaciais.

Para a realização deste estudo, o paciente consentiu e assinou o termo de consentimento livre e esclarecido (TCLE) autorizando a descrição do seu caso, que envolveu a reabilitação com prótese parcial removível de recobrimento "overlay" associada ao obturador faríngeo.

Durante o atendimento ambulatorial no centro especializado de tratamento de anomalias craniofaciais, foi observada presença de maxila atrésica, ausências dentárias múltiplas e colápso máxilo-mandibular (Figura 1). Paciente já utilizava uma prótese parcial removível de recobrimento provisória com substituição dos dentes ausentes, mas apresentava queixas em relação à fala.

Paciente passou por avaliação da equipe interdisciplinar para definição de conduta em relação às alterações de fala, com

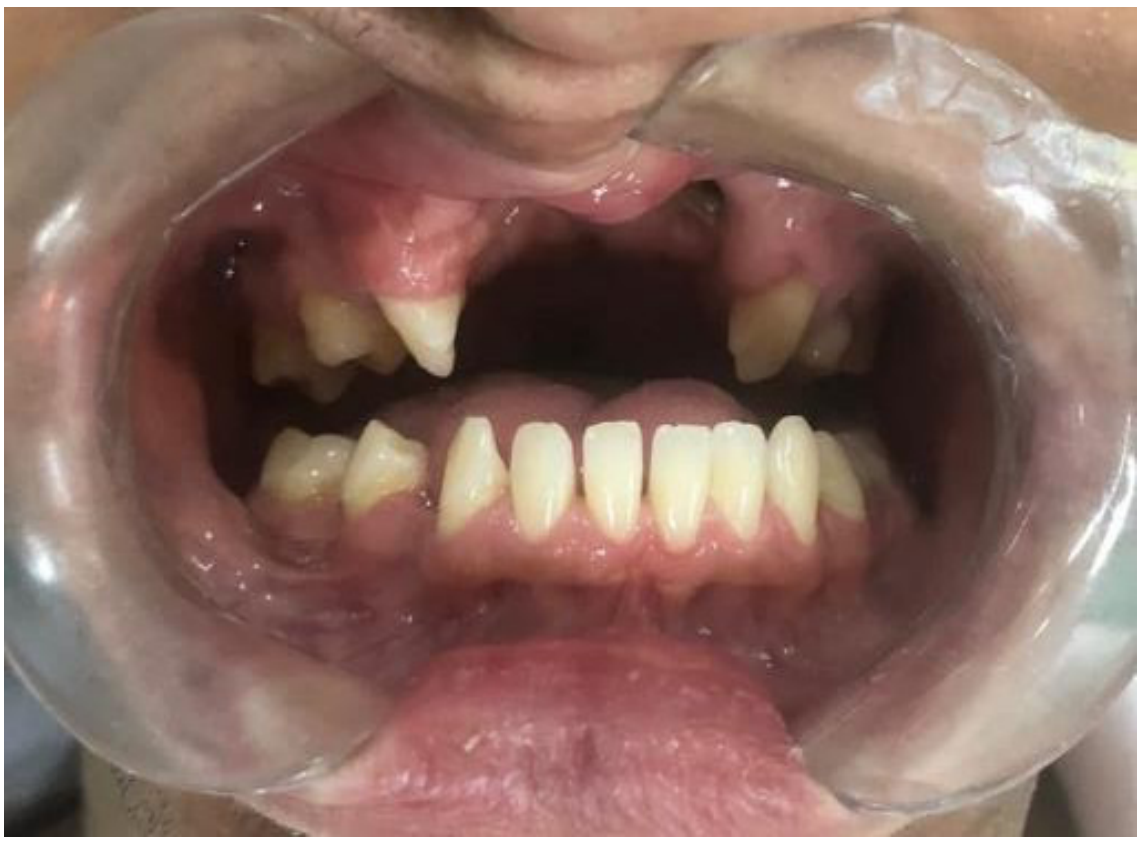

FIGURA 1 - Vista frontal. Condição intraoral prévia ao tratamento. 
diagnóstico fonoaudiológico de insuficiência velofaríngea e presença de articulação compensatórias, necessitando de reabilitação anatômica e funcional da fala, por meio de obturador faríngeo associado à fonoterapia. Neste caso, tanto o cirurgião plástico, como o fonoaudiólogo, contraindicaram a realização de uma nova cirurgia para reabilitação deste mecanismo, por apresentar gap velofaríngeo extenso.

Em relação à avaliação odontológica inicial, foi observado que os dentes pilares naturais do paciente, utilizados como retentores, se apresentavam muito expulsivos, devido ao mau posicionamento e desalinhamento dentário, o que prejudicava a retenção da prótese, seguindo os princípios básicos da prótese parcial removível.

Visando a efetividade do tratamento, foi necessária a criação de novas formas de retenção para os dentes pilares, o delineamento do modelo de gesso e o enceramento dos dentes pilares permitiu o ajuste do eixo de inserção. Os dentes 23 e 26 foram preparados para receberem coroas metálicas fresadas, e os dentes $13 \mathrm{e}$ 26 também foram preparados para receberem coroas metálicas, porém, foram unidas por uma barra metálica criando um sistema barra-clipe sobre dentes (Figuras $2 \mathrm{e} 3$ ), permitindo melhora da estabilidade do aparelho, haja visto o peso do aparelho será aumentado com o acréscimo da extensão do bulbo para disfunção velofaríngea. As coroas metálicas em conjunto com a prótese parcial removível de recobrimento "overlay" possibilitaram ao paciente condições funcionais e estéticas, como mostrado na Figura 4. Após a finalização da prótese (Figura 5) o paciente foi encaminhado para o setor de prótese de palato.

A confecção do obturador faríngeo para disfunção velofaríngea foi realizada de forma gradual, ou seja, por etapas. A primeira etapa foi realizada uma extensão tangenciando o palato mole, encerada pelo cirurgião-dentista, e posteriormente acrilizada, reforçada com um fio metálico. Esta extensão é denominada didaticamente como "porção intermediária" do obturador faríngeo e deve permanecer passiva em relação ao palato mole ${ }^{12,16}$. 


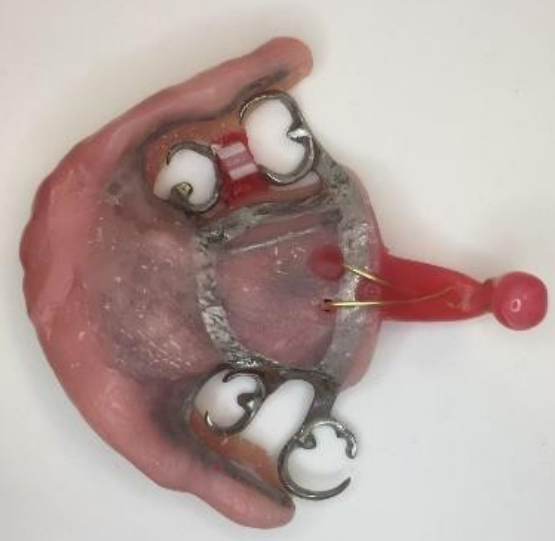

FIGURA 6 - Vista interna do enceramento da etapa do intermediário e do pré-bulbo.

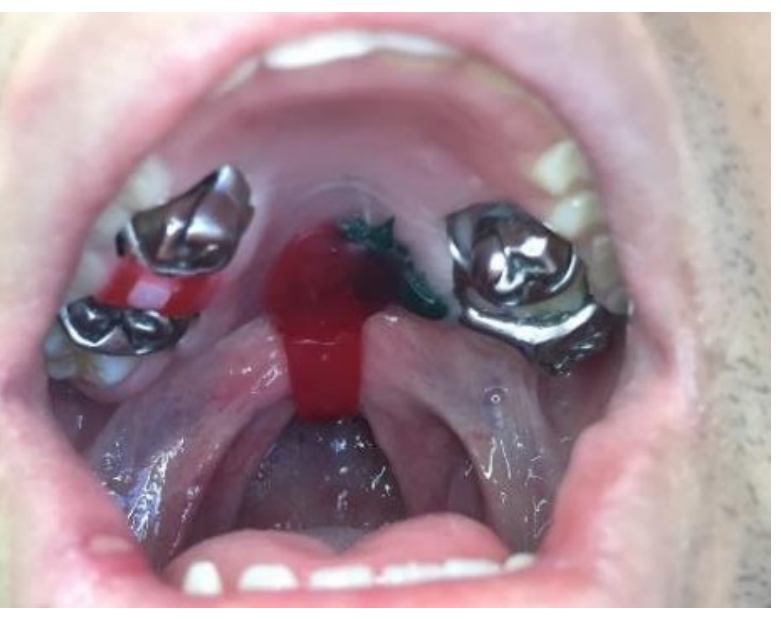

FIGURA 8 - Posicionamento do enceramento do intermediário no palato mole.

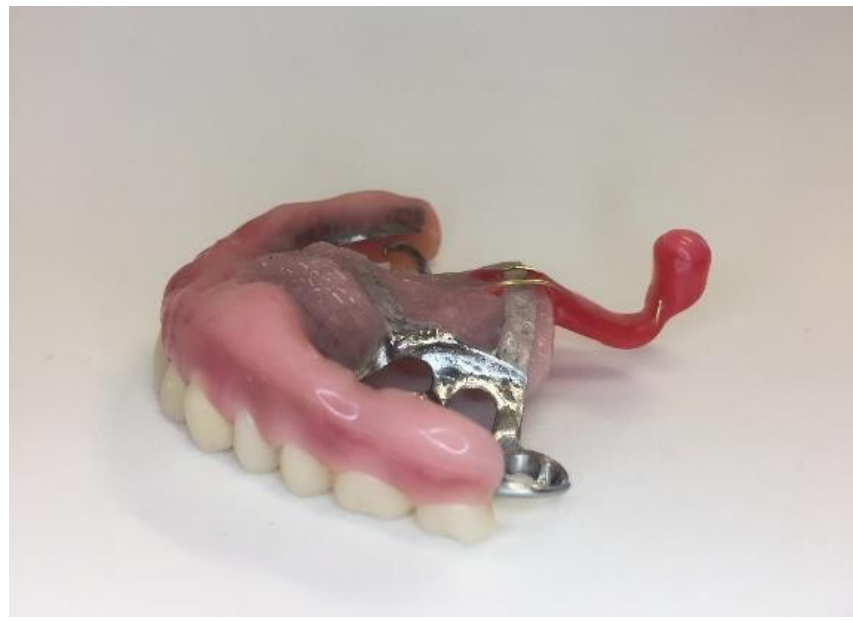

FIGURA 7 - Enceramento da etapa do intermediário e do pré-bulbo.

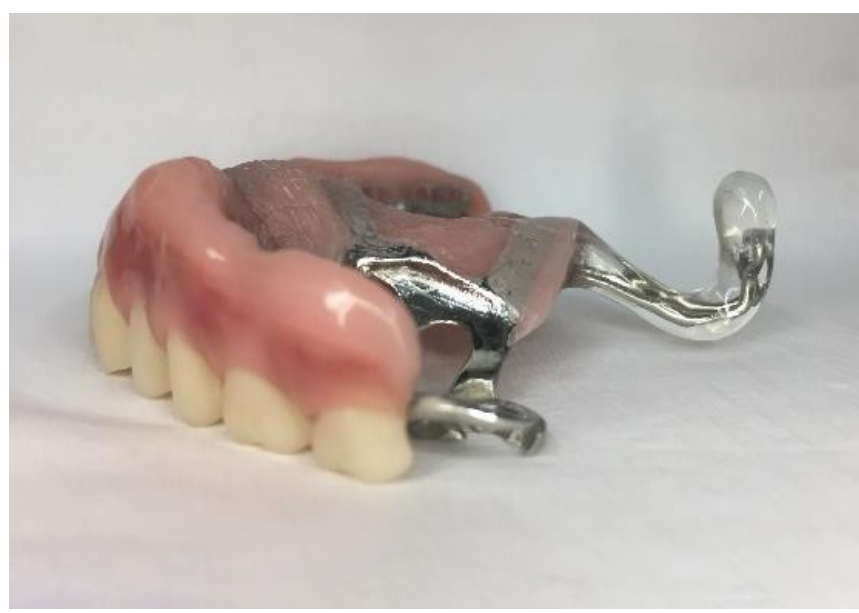

FIGURA 9 - Intermediário e pré-bulbo acrilizados com reforço em metálico.

Após adaptação e o uso efetivo da prótese pelo paciente com a porção intermediária e o pré-bulbo acrilizados (Figuras 9 e 10), foi realizada a moldagem do obturador faríngeo, propriamente dito. O material de moldagem escolhido foi a godiva, uma vez que este material termoplastico é capaz de imprimir as reestrâncias da musculatura da faringe, durante a fala. Esta moldagem foi realizada em cadeira odontológica com o auxílio do fonoaudiólogo, para que fosse avaliada a fala do paciente durante o procedimento de moldagem e após esta, verificando seus efeitos imediatos. O objetivo do fonoaudiólogo foi solicitar a produção dos fonemas orais, a partir de terapia diagnóstica, de 
função velofaríngea, por meio de espelho de Glatzel (Figura 13), verificando o posicionamento do obturador faríngeo e seu efeito imediato sobre a fala. O cirurgião-dentista, por sua vez, avaliou a presença de toques indevidos durante o repouso e durante os movimentos rotacionais da cabeça, por meio de testes com evidenciadores de contato e enquanto o paciente realizava movimentos rotacionais da cabeça e pescoço com grande amplitude ${ }^{12,16}$.

\section{Discussão}

Uma das prioridades do atendimento de indivíduos com fissura labiopalatina é a correção da malformação por meio dos procedimentos cirúrgicos primários. Em muitos centros, estes procedimentos são realizados no primeiro ano de vida do bebê, concomitante ao início do processo de aquisição de fala e linguagem, porém já restringindo o potencial de crescimento facial ocasionado pelas inevitáveis cicatrizes. Desta forma, apesar da reabilitação de indivíduos com fissura labiopalatina ser pautada em protocolos internacionais, as alterações anatomofuncionais, consequentes dos procedimentos cirúrgicos, deverão ser tratadas em época oportuna.

Em relação à estética oclusal, segundo Mazaheri ${ }^{17}$ (1962), 95\% dos pacientes, cuja fissura envolve o rebordo alveolar, necessitaram de algum tipo de prótese, e dessa forma, o tratamento odontológico se torna fundamental. As características benéficas com as sobredentaduras são: melhor dissipação de forças oclusais, preservação do rebordo ósseo, aumento da eficiência mastigatória, estabilidade da prótese, manutenção da propriocepção, devido à presença do ligamento periodontal das raízes dos dentes remanescentes, e a melhor aceitação psicológica por parte do paciente ${ }^{18-22}$.

A prótese parcial removível de recobrimento tem sido grande aliada dos protesistas pela sua versatilidade, pois possibilita diferentes desenhos e dinamismo de uso da resina acrílica em 
diferentes locais, fixada a armação metálica que proporciona rigidez e retenção. Estudos relatam que o sucesso da prótese depende de um bom controle, e uma higiene adequada, para que não ocorram perdas dentárias ${ }^{23-27}$. É importante que o dentista oriente o paciente sobre os efeitos da prótese na cavidade oral, e que esta requer adaptação, inclusive durante a alimentação e a fala. Os cuidados relacionados à prótese interferem na durabilidade da mesma, sendo assim, é necessário cuidado do paciente com sua higiene oral, atentando-se ao uso de fio dental pelo menos uma vez por dia e escovação após as principais refeições. A prótese deve ser removida ao escovar e ao dormir. Além disso, deve-se realizar retornos periódicos ao dentista para avaliação da estabilidade devido a flexibilidade dos grampos e reabsorção do osso/possíveis alterações na área em que a prótese é apoiada. Esse tipo de prótese pode contribuir para uma melhora na estética do perfil facial e volume dos lábios do paciente, alterando em muitos casos aspectos do convívio social e proporcionando maior qualidade de vida ao usuário.

O tratamento da disfunção velofaríngea é primordial para a melhora da qualidade de vida, uma vez que a comunicação oral é parte do convívio social, tanto no âmbito familiar, escolar, de trabalho, entre outros. A disfunção velofaríngea, pode prejudicar todo o trato vocal, uma vez que podem apresentar maior esforço respiratório, laríngeo e articulatório, na tentativa de compensar a deficiência de pressão intraoral, que prejudica a pressão subglótica, a intensidade vocal e a precisão articulatória $^{2}$. O obturador faríngeo, associado à fonoterapia, tem sido uma alternativa de tratamento para pacientes com contraindicação de outras cirurgias no palato ou faringe, a ser definida pela equipe especializada. O obturador faríngeo tem a função de auxiliar o controle de fluxo de aéreo, durante a fala, uma vez que obtura o gap velofaríngeo, por meio do toque das estruturas envolvidas na extensão posterior acrílica. 
O processo de confecção da prótese deste estudo foi realizada com a participação de uma equipe transdisciplinar especializada, composta por cirurgião- dentista e fonoaudiólogo. Por isso, semelhante ao processo de confecção e adaptação da uma prótese parcial removível, o obturador faríngeo também requer cuidados e tempo de adaptação em relação de etapas e sequência de atendimentos ${ }^{16}$. Além disso, a cada etapa, o paciente deve se sentir seguro e confortável, tanto durante o repouso, quanto durante a alimentação e a fala. A adaptação do paciente para o uso deste tipo de prótese durante a alimentação requer atenção especial da equipe, pois o ambiente das refeições, geralmente se enquadram em encontros sociais que resultam também em comunicação oral, cuja reabilitação da disfunção velofaríngea é envolvida ${ }^{16}$.

Para a manutenção da prótese com obturador faríngeo os controles periódicos semestrais são sugeridos, e alguns fatores devem ser checados por parte do ortodontista, Protesista ou Odontopediatra. Primeiramente, os grampos devem estar bem adaptados aos dentes retentores, por esta razão o enceramento das coras metálicas devem ser executados com o auxílio do delineador permitindo um correto eixo de inserção. A parte acrílica anterior da prótese deve ser observada, pois caso haja fratura ou trinca a mesma deve ser corrigida com moldagem com a prótese em uso (na cavidade oral) e enviada ao laboratório ${ }^{15}$. Além disso, é muito importante a observação de toda a cavidade oral e atenção aos relatos de desconforto do paciente, para que eventuais lesões devido à compressão da porção intermediária da prótese em tecido do palato mole, que não deve apresentar alteração de coloração ou marcas de compressão com ou sem ulceração. O acúmulo de secreção em parede posterior da velofaringe pode ser resultado deslocamento do bulbo devido a desajustes dos grampos ${ }^{15}$.

A literatura mostra que o tratamento da disfunção velofaríngea é constituído por procedimentos físicos para a correção da 
insuficiência velofaríngea (cirurgia ou obturador faríngeo) e por procedimentos funcionais para a correção dos erros de aprendizagem (fonoterapia), sendo comum que um mesmo paciente de um procedimento físico associado à fonoterapia ${ }^{28,29}$. Por este motivo, uma vez que a confecção da prótese associada ao obturador faríngeo foi concluída e houve boa adaptação do paciente, o mesmo é orientado a realizar terapia fonoaudiológica para que a reabilitação funcional ocorra. O objetivo é propiciar a contração muscular das estruturas envolvidas no mecanismo velofaringeo, a partir do controle pressórico intraoral para a produção de sons orais, compatíveis com a língua portuguesa, corrigindo as compensações articulatórias ${ }^{30,16}$. Durante o processo terapêutico é importante à realização de avaliações periódicas da fala desse paciente, pois em casos onde não haja melhora na inteligibilidade de fala entre o atendimento atual e o anterior o paciente deve ser encaminhado ao cirurgião-dentista para checar alterações na estrutura e posicionamento da prótese e a efetividade da abordagem fonoaudiológica ${ }^{16}$.

No caso deste trabalho, o paciente apresentava maxila atrésica, que pode ter sido consequência da deficiência de crescimento facial induzido pelas cirurgias de primárias ${ }^{5-9}$. Assim como a insuficiência velofaríngea, também pode ter sido sequela da cirurgia de palatoplastia primária, permanecendo as alterações de fala, como a hipernasalidade e as compensações articulatórias.

Pinto $^{28}$ (2016) e Ferreira ${ }^{29}$ (2018) descrevem, em seus estudos, resultados satisfatórios de pacientes que utilizavam obturadores faríngeos e foram submetidos ao processo de reabilitação funcional, por meio de fonoterapia. Em ambos os estudos, foi observada melhora na inteligibilidade de fala, na maioria dos casos avaliados. Este estudo de caso teve como limitação, a não avaliação dos resultados de fala após o processo de reabilitação fonoaudiológica, no qual o paciente foi orientado a realizar após a conclusão da confecção da prótese dentária associada ao obturador faríngeo, sendo que este não foi o objetivo deste estudo. 
Muitos trabalhos referem que indivíduos com fissura labiopalatina podem apresentar alterações oclusais, discrepância maxilomandibular, alterações de fala e, até acometimento psicológico. Porém a literatura é escassa, no que diz respeito à reabilitação global do indivíduo, envolvendo a equipe interdisciplinar, e a interação entre cirurgião-dentista e fonoaudiólogo, para tal. Este estudo trouxe uma visão holística de tratamento individualizado, porém, na qual a transdisciplinariedade foi aplicada, visando à melhora estética e funcional, das possíveis sequelas de um indivíduo com fissura labiopalatina.

\section{Conclusão}

Este estudo trouxe a caracterização das etapas de tratamento protética de um paciente com fissura de labio e palato bilateral operado, porém com sequelas na estética e na fala, por meio da reabilitação oral com uma prótese parcial removível de recobrimento "overlay" associada ao obturador faríngeo. Notou-se que a equipe interdisciplinar de cirurgiões-dentistas e fonoaudiólogos preconizaram, não só a melhora estética, mas também funcional, visando a melhora da qualidade de vida deste indivíduo. Enfatizase que a reabilitação da disfunção velofaríngea dependerá do processo de fonoterapia, o qual o paciente foi orientado a realizar.

\section{Referências}

1 - Montagnoli LC, Barbieri MA, Bettiol H, Marques IL, Souza LD. Prejuízo no crescimento de crianças com diferentes tipos de fissura lábio-palatina nos 2 primeiros anos de idade: um estudo transversal. Jornal de Pediatria. 2005; 81(6): 461-5.

2- Silva P et al. Aspectos Patofisiológicos do Esfincter Velofaríngeo nas Fissuras Palatinas. Arq Int Otorrinolaringol / Intl Arch Otorhinolaryngol. 2008;12: 426-435.

3 - Collares MV, Westphalen AC, Costa TC, Goldim JR. Fissuras lábiopalatinas: incidência e prevalência da patologia no Hospital de Clínicas de Porto Alegre: um estudo de 10 anos. Rev Amrigs. 1995; 39(3):183-8.

4 - Semb G. A study of facial growth in patients with bilateral cleft lip and palate treated by the Oslo CLP Team. The Cleft Palate-Craniofacial Journal. 1991; 28(1): 22-39. 
5 - Pinto JHN, Lopes JFS. Reabilitação oral com prótese dentária. In: Trindade IEK, Silva Filho OG. Fissuras labiopalatinas: uma abordagem interdisciplinar. São Paulo: Santos; 2007. p. 261-74

6 - Semb G. A study of facial growth in patients with unilateral cleft lip and palate treated by the Oslo CLP team. The Cleft Palate-Craniofacial Journal. 1991; 28(1): 1-21.

7- Silva Filho OG, Ramos AL, Camargo ARC. The influence of unilateral cleft lip and palate on maxillary dental arch morphology. The Angle Orthodontist. 1992; 62(4): 283-90.

8 - Capelozza FL, Almeida AM, Ursi WJ. Rapid maxillary expansion in cleft lip and palate patients. Journal of clinical orthodontics: JCO. 1994; 28(1): 34-39.

9- Heidbuchel KL, Kuijpers-Jagtman AM, Van't Hof MA, Kramer GJ, Prahl-Andersen B. Effects of early treatment on maxillary arch development in BCLP. A study on dental casts between 0 and 4 years of age. Journal of Cranio-Maxillofacial Surgery. 1998; 26(3): 140-7.

10 - Rizoto A, Ribeiro MC, Ferreira AR, Cembraneli TZ, Lopes JF, Pinto $\mathrm{JH}$. Reabilitação de paciente portador de fissura através de prótese de recobrimento: relato de caso clínico. PCL. 2000; 2(5): 22-6.

11 - Cardia CC, Yamashita RP, Campos LD, Sampaio-Teixeira AC, Trindade-Suedam IK, Trindade IE. Obstrução respiratória após cirurgia de retalho faríngeo para correção de insuficiência velofaríngea: revisão da literatura. Rev Bras Cir Craniomaxilofac. 2011; 14(4): 207-13.

12 - Pegoraro-Krook MI, Souza JCRD, Magalhães LCT, Feniman MR. Intervenção fonaudiológica na fissura palatina. In: Ferreira LP, BefiLopes DM, Limongi SCO (Orgs.). Tratado de fonoaudiologia. São Paulo: Roca; 2004. p. 439-55.

13 - Marrinan E, Shprintzen RJ. Cleft palate and craniofacial disorders. In: Anderson NB, SHAMES, GH. Human communication disorders. An introduction.7thed.United States of America: Pearson Education; 2006. p. 263-278.

14 - Smith BE, Kuehn DP. Speech evaluation of velopharyngeal dysfunction. Journal of Craniofacial Surgery. 2007; 18(2): 251-61.

15 - Santos JA, Dutka JCR, Lopes MMW. Manutenção da prótese de palato: fluxograma de orientação para cirurgiões-dentistas [Trabalho de Conclusão da Residência Multiprofissional em Síndromes e Anomalias Craniofaciais]. Bauru: Hospital de Reabilitações de Síndromes e Anomalias Craniofaciais; 2019.

16 - Pegoraro-Krook MI, Aferri HC, Uemeoka E. Prótese e obturadores faríngeos. In: Jesus MSV, DI NINNO CQMS. Fissuralabiopalatina: fundamentos para a prática fonoaudiológica. São Paulo: Rocca; 2009.

17 - Mahazeri M. Indications and contraindications for prosthetic speech appliances in cleft palate. Plastic and Reconstructive Surgery. 1962; 30(6): 663-9. 
18 - Duckmanton NA. The role of the overlay denture in the prevention of mandibular ridge atrophy. Australian dental journal. 1978; 23(4): 332-43.

19 - Bolouri A. Proposed treatment sequence for overdentures. The Journal of Prosthetic Dentistry. 1980; 44(3): 247-50.

20 - Renner RP, Gomes BC, Shakun ML, Baer PN, Davis RK, Camp P. Fouryear longitudinal study of the periodontal health status of overdenture patients. The journal of prosthetic dentistry. 1984; 51(5): 593-8.

21 - Kaniff AA, Sommerfeld RM. A case presentation of maxilary immediate denture opposing a mandibular overdenture. Ill Dent J. 1985; 54(2): 84-7.

22 - Loiselle RJ, Crum RJ, Rooney GE, Stuever CH. The physiologic basis for the overlay denture. Journal of Prosthetic Dentistry. 1972; 28(1): 4-12.

23 - Dolder EJ. The bar joint mandibular denture. J Prosth Dent. 1961; 11(4): 689-707.

24 - Robins JW. Success of overdentures and prevention of failure. J Am Dent Ass. 1980; 100(6): 858-62.

25 - Ettinger RL. Tooth loss in overdenture population. J Prosth Dent. 1988; 60(4): 459-62.

26 - Gennari Filho H, Silva Filho CE. De overdenture. Rev. Bras Odontol. 1994; 51(82): 44-7.

27 - Langer Y, Langer A. Root-retained overdentures. Part 11: managing trauma between edentulous ridges and opposing dentition. J Prosth Dent. 1992; 67(1):77-81

28 - Pinto MDB. Resultados da fonoterapia intensiva para correção da oclusiva glotal e fricativa faríngea na fissura labiopalatina. 2016. Tese (Doutorado em fissuras Orofaciais) - Hospital de Reabilitação de Anomalias Craniofaciais, Universidade de São Paulo; Bauru; 2016.

29 - Ferreira GZ. Programa de fonoterapia intensiva em pacientes com fissura labiopalatina. 2018. Tese (Doutorado em Fonoaudiologia) Faculdade de Odontologia de Bauru, Universidade de São Paulo; Bauru; 2018.

30 - Trindade IE, Genaro KF, Yamashita RP, Miguel HC, Fukushiro AP. Proposta de classificação da função velofaríngea na avaliação perceptivo-auditiva da fala. Pró-Fono Revista de Atualização Científica. 2005; 17(2): 259-62. 


\title{
Prosthetic rehabilitation of maxillary atresia and velopharyngeal dysfunction: a case report
}

\begin{abstract}
The cleft lip and palate involve the region of the alveolar ridge which can cause anomalous development of the teeth in the regions of the gap that alter the dental alignment compromising the aesthetics of the smile. In addition, surgeries for its correction may have restrictive effects on the development of the maxilla bringing changes in the middle third of the face including maxillary atresia. This can lead to occlusal instability, unsatisfactory aesthetics, phonetic changes and mouth breathing. Once the cleft palate surgeries have not been completely successful the individual may experience velopharyngeal dysfunction. This compromises the balance of resonance between the nasal and oral cavities causing changes in speech such as: articulatory compensations and hypernasality. The purpose of this study is to describe the prosthetic rehabilitation of a 26-year-old male patient with trans-foramen bilateral cleft, incisional diagnosis of maxillary atresia and velopharyngeal dysfunction due to post-surgical sequelae. In order to rehabilitate the aesthetics and oral functions of this individual a partial removable overlay prosthesis was made retained by milled metal crowns on teeth 23 and 26 and metal crowns on teeth 13 and 16, joined by a bar and use bar-clip system to improve its retention. For speech rehabilitation a pharyngeal obturator associated with dental prosthesis has been proposed. It is concluded that this treatment provided an improvement in their aesthetics as well as the functions of eating/swallowing and speech, also providing an improvement in the individual's self-esteem and quality of life.
\end{abstract}

KEYWORDS: Cleft lip. Cleft palate. Velopharyngeal Insufficiency.

\section{Como citar este artigo}

Lopes JFS, Rabelo ALL, Lopes MMW, Amaral AM, Pinto MDB, Whitaker ME. Reabilitação protética da maxila atrésica e da disfunção velofaríngea: relato de caso clínico. Rev Odontol Bras Central 2021; 30(89): 387-402. DOI: 10.36065/robrac.v30i89.1408 